Revista Brasileira de Meteorologia, v.25, n.4, 448 - 454, 2010

\title{
ESTIMATIVA DO COEFICIENTE PRIESTLEY-TAYLOR EM FLORESTA MONODOMINANTE CAMBARAZAL NO PANTANAL
}

\section{LUCIANA SANCHES ${ }^{1}$, MARCELO DE CARVALHO ALVES ${ }^{2}$, JOSÉ HOLANDA CAMPELO JÚNIOR ${ }^{2}$, JOSÉ DE SOUZA NOGUEIRA ${ }^{3}$, HIGO JOSÉ DALMAGRO²}

\author{
${ }^{1}$ Departamento de Engenharia Sanitária e Ambiental, Universidade Federal de Mato Grosso (UFMT) \\ ${ }^{2}$ Departamento de Solos e Engenharia Rural, Universidade Federal de Mato Grosso (UFMT) \\ ${ }^{3}$ Departamento de Física, Universidade Federal de Mato Grosso (UFMT)
}

lsanches@ufmt.br, mdecalves@ufmt.br, campelo@ufmt.br, nogueira@ufmt.br,higo@ufmt.br

Recebido Setembro 2009 - Aceito Fevereiro 2010

\begin{abstract}
RESUMO
O coeficiente Priestley-Taylor ( $\alpha$ ) foi calculado baseado na estimativa da evapotranspiração pelo método de Bowen para floresta monodominante Vochysia divergens no Pantanal, Brasil. A área em estudo estava localizada no noroeste do Pantanal a aproximadamente 160 km de Cuiabá, Mato Grossso, Brasil (16 39'50"'S; 56 47'50"O). Medições micrometeorológicas contínuas, em uma torre a uma altura de $32 \mathrm{~m}$ de altura, foram feitas de janeiro a dezembro de 2007. A evapotranspiração variou de 2,50 $\mathrm{mm} \mathrm{dia}^{-1}$ (estação seca) a 4,10 $\mathrm{mm} \mathrm{dia}^{-1}$ (estação úmida). O coeficiente Pristley-Taylor $(\alpha)$ variou durante o ano com valores máximos e mínimos nas estações seca e úmida, respectivamente, com média anual de $0,65 \pm 0,18$ de acordo com o padrão climático da área em estudo em função do aumento do conteúdo de água no solo/lâmina d'água de inundação e/ou diminuição na demanda evaporativa. Com a determinação empírica das dimensões de $\alpha$, as estimativas da evapotranspiração podem ser melhoradas para florestas de Vochysia divergens na planície de inundação do Pantanal.

Palavra-chave: evapotranspiração, inundação, sazonalidade
\end{abstract}

\begin{abstract}
ESTIMATION OF THE PRIESTLEY-TAYLOR COEFFICIENT IN THE MONOSPECIFIC FOREST IN NORTHERN PANTANAL, BRAZIL .

The Priestley-Taylor coefficient $(\alpha)$ was calculated based on the Bowen method evapotranspiration estimative for Vochysia divergens monospecific forests in Pantanal, Brazil. The study area was located at the northeastern Pantanal, approximately $160 \mathrm{~km}$ from Cuiabá city, Mato Grosso State, Brazil $\left(16^{\circ} 39^{\prime} 50^{\prime \prime} \mathrm{S} ; 56^{\circ} 47^{\prime} 50^{\prime \prime} \mathrm{W}\right)$. Continuous micrometeorological flux measurements at a $32 \mathrm{~m}$ tower height were made from January to December of 2007. The evapotranspiration ranged from $2.26 \mathrm{~mm}$ day $^{-1}$ (dry season) to $4.50 \mathrm{~mm} \mathrm{day}^{-1}$ (wet season). The average estimated $\alpha$ value ranged along the year from a maximum and a minimum during the dry and wet season respectively, with an annual average of $0.65 \pm 0.18$ according to the weather pattern over the study area due to the increase of water content in soil/water flooding depth and/or decrease in evaporative demand. Based on empirical $\alpha$ values, estimates of evapotranspiration can be improved for Vochysia divergens forests on Pantanal floodplain.
\end{abstract}

Keywords: evapotranspiration, floodplain, seasonality.

\section{INTRODUÇÃO}

O Pantanal é uma planície quaternária com mais de $160.000 \mathrm{~km}^{2}$, que originou a planície da região da bacia do Alto Rio Paraguai. Em território nacional possui 138.183 $\mathrm{km}^{2}$, com $65 \%$ do seu território no estado de Mato Grosso do Sul e 35\% no Mato Grosso (Castelnou et al., 2003). O Pantanal brasileiro tem muitos tipos de composição florestal sujeitas à inundação. Muitas delas são monodominantes, assim como a vegetação de relevância, Vochysia divergens, Vochysiaceae (cambará), que vigorosamente se espalha em pastagens e forma um padrão monoespecífico chamado 
localmente "cambarazais". Vochysia divergens é uma árvore que cresce entre 28 - 30m e tem uma coroa de folhas densas com folhagem simples. No entanto, esta vegetação pode ser influenciada por variáveis meteorológicas, como a evapotranspiração.

A evapotranspiração pode ser estimada em função de vários parâmetros e numerosos métodos (Penman, 1948, Thornthwaite, 1948, Priestley e Taylor, 1972; Hargreaves, 1994).

A equação de Priestley e Taylor (1972) é um dos métodos mais comumente empregados (Xiaoying e Erda, $2005)$, nela se emprega o coeficiente de Priestley-Taylor $(\alpha)$ que em resumo, representa o controle evaporativo efetuado pela vegetação e é definido como $\alpha=\mathrm{E} / \mathrm{E}_{\text {eq }}$, em que E é a taxa de evaporação e $\mathrm{E}_{\mathrm{eq}}$ é a taxa de evaporação em equilíbrio (Priestley e Taylor, 1972; Raupach, 2001). Alguns métodos modificados estimam $\alpha$ em função de variáveis ambientais. Entretanto, nenhum é considerado um método único e funcional para determinação de $\alpha$ e deve ser determinada empiricamente (Sumner e Jacobs, 2005). O método de Priestley-Taylor despreza a influência do déficit de pressão de vapor na evapotranspiração de referencia, assumindo que a ET depende somente da radiação solar e da temperatura do ar. A estimativa da evapotranspiração pelo método de Pristey-Taylor tem sido objeto de discussão devido aos diferentes tipos de cobertura vegetal. Neste contexto, o presente trabalho teve como objetivo estimar o coeficiente Pristley-Taylor $(\alpha)$ com base na evapotranspiração estimada pela razão de Bowen em florestas monodominantes Vochysia divergens no Pantanal Mato-Grossense, Brasil.

\section{MATERIAL E MÉTODOS}

\subsection{Localização e Descrição da Área em Estudo}

O estudo foi conduzido no nordeste do Pantanal, aproximadamente $160 \mathrm{~km}$ de Cuiabá ou $60 \mathrm{~km}$ de Poconé, Mato Grosso, Brasil (16³9’50”S e 5647’50”O), conforme Figura 1. Esta é uma área pertencente a Reserva Particular do Patrimônio Natural - RPPN SESC - Pantanal, composta por vegetação monodominante de Cambará (Vochysia divergens), conhecida localmente como Cambarazal, com altura do dossel variando entre 28 a $30 \mathrm{~m}$ e solo classificado como GLEISSOLO HÁPLICO Ta Distrófico típico.

O clima é caracterizado por uma estação seca pronunciada e estação chuvosa, Aw segundo classificação climática de Köppen. A precipitação média anual é de aproximadamente 1.400 milímetros com precipitação máxima em janeiro e mínima em julho. Inundações seguem a estação chuvosa, devido à paisagem plana, o padrão de inundação no interior do pantanal é fortemente influenciado pela precipitação local. Nesta área, conhecida como Pantanal do município de Poconé, as inundações são bastante superficiais, até cerca de $2 \mathrm{~m}$ de profundidade. Durante o período de água baixa, devido as planícies baixas, muitos dos lagos de várzea são desconectados do canal do rio (Nunes da Cunha e Junk, 2004).

\subsection{Medição das Variáveis Micrometeorológicas}

Medições contínuas de variáveis meteorológicas foram realizadas por sensores instalados em uma torre de 32 metros de

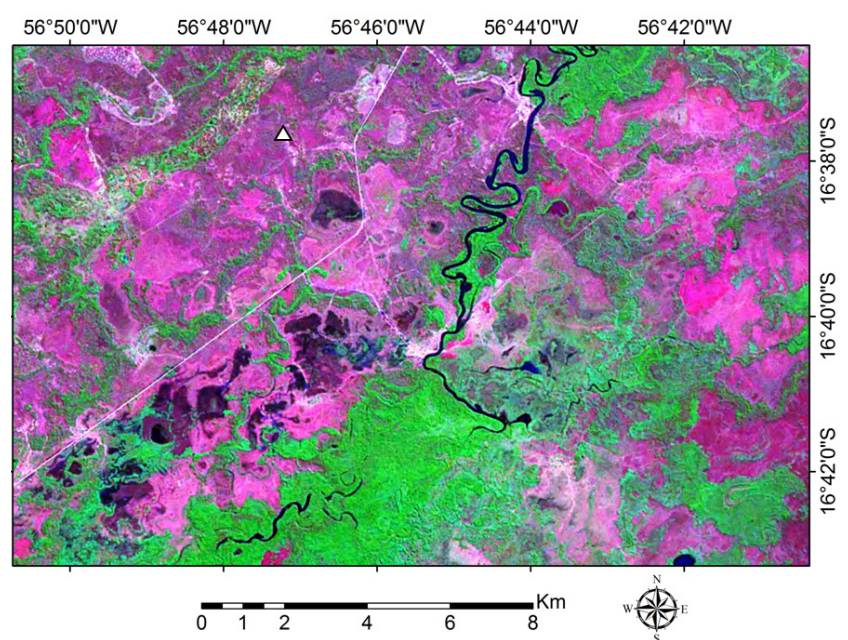

Figura 1-. Área em estudo aproximadamente a $60 \mathrm{~km}$ da cidade de Cuiabá, Mato Grosso, Brasil $(\Delta)$, coordenada da torre meteorológica $16^{\circ} 39$ '50”S e 56²'50”O (Landsat ETM+ GeoCoverTM 2000 imagem). 
altura entre janeiro a dezembro de 2007. O saldo de radiação foi medido por meio de um sensor (Net Radiometer Kipp \& Zonen Delft Inc. Holland ${ }^{\circledR}$ e de um piranômetro LI-200 Campbell Sci Inc. USA ${ }^{\circledR}$ ) instalado a $33 \mathrm{~m}$ de altura. A temperatura e a umidade relativa do ar foram medidos por meio de dois termohigrômetros (HMP 45 C Vaisala Inc. Helsinki Finland $\AA$ ) instalados nas alturas de 33,7 m e 37,7 m. Este termohigrômetro mede a umidade relativa do ar em um intervalo de 0 a $100 \%$, com precisão de $\pm 2 \%$ UR ( 0 a $90 \%$ UR) e $\pm 3 \%$ UR ( 90 a 100 $\%$ UR), e temperatura do ar em um intervalo de -40 a $+60^{\circ} \mathrm{C}$ e precisão de $\pm 0,55^{\circ} \mathrm{C}$. Adjacente a torre micrometeorológica foi instalada uma placa de fluxo de calor no solo (G) (HFT-3.1 REBS Inc. Seattle Washington $\left.{ }^{\circledR}\right)$ a $2 \mathrm{~cm}$ de profundidade.

Os sensores micrometeorológicos e de medição de fluxo de calor no solo estavam conectados a um sistema de controle e registro acoplado a um multiplexador (AM 16 Cambpell Scientific Inc. Logan UT USA ${ }^{\circledR}$ ), alimentado por um conjunto de baterias mantidas carregadas por meio de painéis solares (SP 65 Cambpell Scientific Inc. Logan UT USA () ). Os dados foram coletados e armazenados a cada 15 minutos em um datalogger (MM900 ELE International Bedfordshire UK ${ }^{\circledR}$ ) e transferidos para o disco rígido de um computador a cada quinze dias.

Devido ao mau comportamento dos registros obtidos pelo pluviômetro instalado na torre micrometeorológica, foram utilizados os dados de precipitação medidos no posto da RPPN Sesc Pantanal de Porto Cercado distante aproximadamente 15 $\mathrm{km}$ da torre micrometeorológica, mesmo local de medição da altura da lâmina d'água.

Para a determinação do perfil vertical da temperatura do ar e da umidade relativa do ar durante a estação úmida, foram utilizadas três estações meteorológicas, modelo 16510SER WeatherLink para Vantage Pro2 - (Serial Port com Datalogger), com sensores de temperatura do ar (intervalo de medida $-32^{\circ} \mathrm{C}$ a $+83^{\circ} \mathrm{C}$ e precisão de $\pm 0,6^{\circ} \mathrm{C}$ ) e a umidade relativa do ar (intervalo de medida 10 a $100 \%$ UR e precisão $\pm 3 \%$ ), instalados nas alturas de 4,8 e $16 \mathrm{~m}$. As estações meteorológicas estavam programadas para fazer leituras das medidas, a cada 30 segundos, com armazenamento a cada 30 minutos, de fevereiro a março de 2009.

Para análise dos resultados, a estação seca foi definida como o número consecutivo com precipitação menor que 117 mm (média mensal da precipitação em 2007), resultando em 6 meses de maio a outubro, e a estação úmida de novembro a abril.

\subsection{Evapotranspiração Estimada pela Razão de Bowen}

A evapotranspiração foi estimada pelo método da razão de Bowen ( $\beta$ ) (Bowen, 1926). A razão de Bowen é a razão entre os fluxos de calor sensível (H) e calor latente (LE), de acordo com a Equação 1 e reescrita pela Equação 2:

$$
\begin{gathered}
\beta=\frac{H}{L E} \\
\beta=\gamma \frac{\Delta T}{\Delta e}
\end{gathered}
$$

onde, $\Delta \mathrm{T}$ é a diferença de temperatura entre dois níveis $\left({ }^{\circ} \mathrm{C}\right), \Delta \mathrm{e}$ a diferença de pressão de vapor d'água entre dois níveis $(\mathrm{kPa}) \mathrm{e}$ $\gamma$ a constante psicométrica $\left(0,0626 \mathrm{kPa}^{\circ} \mathrm{C}^{-1}\right)$. A pressão de vapor de água saturada de ar $\left(\mathrm{e}_{\mathrm{s}}\right)$ em $(\mathrm{kPa})$ para cada altura foi calculada em função da temperatura do ar $\left(\mathrm{T}_{\mathrm{a}}\right)$ em cada altura, de acordo com a Equação 3.

$$
\mathrm{e}_{\mathrm{s}}=0,6108 * 10^{\left(\frac{7,5 \mathrm{~T}_{\mathrm{a}}}{237,3+\mathrm{T}_{\mathrm{a}}}\right)}
$$

A pressão de ar de vapor de água (e) foi calculada de acordo com a Equação 4.

$$
e=e_{s} U R
$$

onde, UR é a umidade relativa do ar (\%).

Com os valores de $\beta$ pode-se estimar o fluxo de calor latente (LE) em $\left(\mathrm{W} \mathrm{m}^{-2}\right)$ pela Equação 5 .

$$
\mathrm{LE}=\frac{\mathrm{Rn}-\mathrm{G}}{(1+\beta)}
$$

onde, Rn é o saldo de radiação líquida e G o fluxo de calor no solo.

\subsection{Estimativa do Coeficiente de Priestley-Taylor $(\alpha)$}

A equação de Priestley-Taylor é uma simplificação da equação de Penman (1948) original, em que somente é contemplado o termo energético corrigido por um coeficiente de ajuste $\alpha$ (Pereira et al., 1997). Por meio de ET conhecida, os valores de $\alpha$ podem ser estimados por meio da equação de Priestley-Taylor reescrita na forma de Equação 6.

$$
\alpha=\mathrm{ET} /[(\mathrm{s} / \mathrm{s}+\gamma)(\mathrm{Rn}-\mathrm{G})]
$$

em que, $\alpha$ é o coeficiente de Priestley-Taylor, s a tangente à curva de pressão de saturação de vapor no ponto médio entre as temperaturas dos psicrômetros e $\gamma$ a constante psicrométrica.

A vantagem do método do Priestley-Taylor é o uso de poucas variáveis para estimar a $\mathrm{ET}$ (Rn, G e temperatura do ar) em relação a outros métodos. A base teórica de $\alpha$ é relativamente bem conhecida, sabendo-se que $\alpha$ pode variar substancialmente, dependendo da rugosidade do dossel e do teor de água de superfície. Priestley e Taylor (1972) determinaram valores $\alpha$ variando de 1,08 (evaporação do solo nu) a 1,32 (evapotranspiração do solo nos vegetais), com valor médio de 1,26 , sendo este aplicado principalmente em climas úmidos. 


\section{RESULTADOS E DISCUSSÕES}

\subsection{Condições Climáticas}

As precipitações máximas ocorreram em novembro e dezembro (estação úmida), enquanto que não houve precipitação em junho, setembro e outubro (Figura 2). A precipitação anual foi aproximadamente $1400 \mathrm{~mm}$, concordando com Hasenack et al. (2003) que mencionaram que a precipitação total média anual no Pantanal apresenta um gradiente Oeste-Nordeste e Leste-Sudeste, em que no Oeste, a precipitação é inferior a 1000 mm e a máxima é aproximadamente $1300 \mathrm{~mm}$.

A inundação iniciou em janeiro finalizando em junho (Figura 2). O pico de inundação ocorreu em março (estação úmida) com menores níveis em maio (estação seca). A variação da inundação está relacionada com a precipitação; sendo que a oscilação anual do nível d'água que ocorre no rio Cuiabá, determina a inundação à jusante na planície do pantanal, sendo altamente influenciada pela precipitação local e difícil drenagem da água da chuva pelo solo (Arieira e Nunes da Cunha, 2006). Em geral, o pulso de inundação anual que ocorre em todo o Pantanal, pode ser descrito como mono-modal com variações temporais e espaciais. Ao longo do canal do rio principal o pulso anual é bem definido, impulsionado principalmente o transbordamento deste rio. Mais distante dos canais principais de escoamento, o pulso de inundação é mais atenuada (Penha et al., 1999) como o ecossistema em estudo.

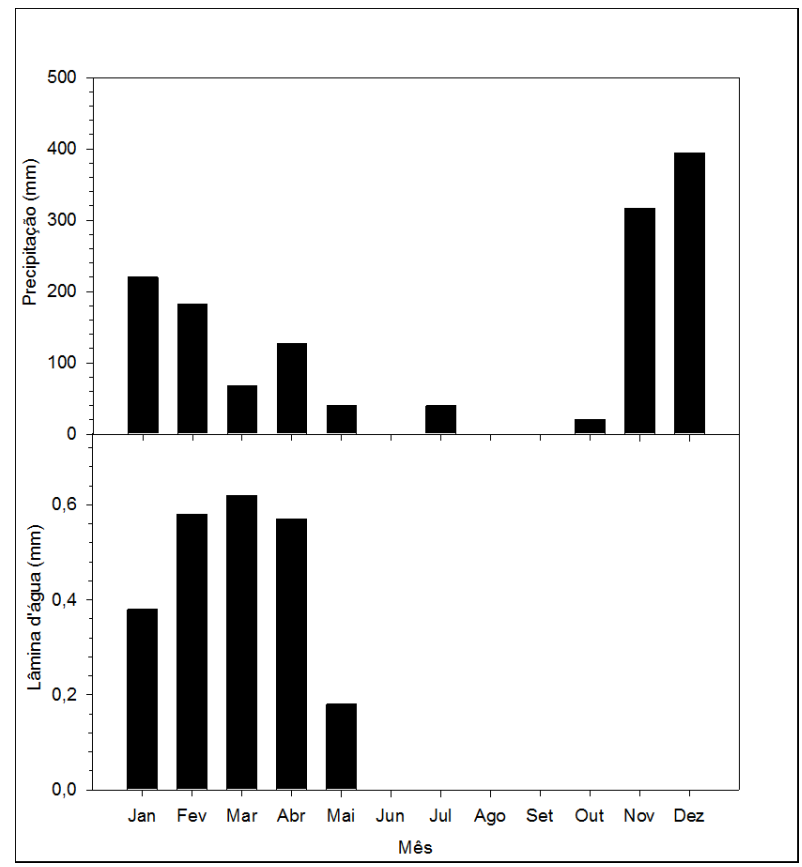

Figura 2 -Precipitação mensal total e lâmina d'água da inundação na área de estudo de janeiro a dezembro de 2007.
A evapotranspiração do ecossistema representa a soma da evaporação à superfície do solo, da folhas durante a troca gasosa e da superfície das plantas após precipitação, neste trabalho, a ET foi estimada por meio da razão de Bowen, sendo 4,1 e 2,5 $\mathrm{mm} \mathrm{d}^{-1}$ durante a estação úmida e seca, respectivamente (Figura 3a). Menores valores de evapotranspiração, durante a estação seca, também foram relatados em Cerrado e floresta de transição Amazônica-Cerrado no Centro-Oeste, enquanto que em florestas tropicais úmidas localizadas em Manaus, Santarém e Rondônia foram relatados menores valores de evapotranspiração durante a estação úmida (Rocha et al., 2009; Miranda et al., 1997). Menores valores de ET, durante a estação seca, podem ser explicados pelas menores temperaturas, $\mathrm{o}$ ar mais seco e menor radiação (solstício de inverno), além de ser uma característica da limitação de água nesta estação neste dossel. Durante a estação seca, ocorre um declínio do conteúdo de água no solo, podendo limitar a evaporação e a disponibilidade de água para

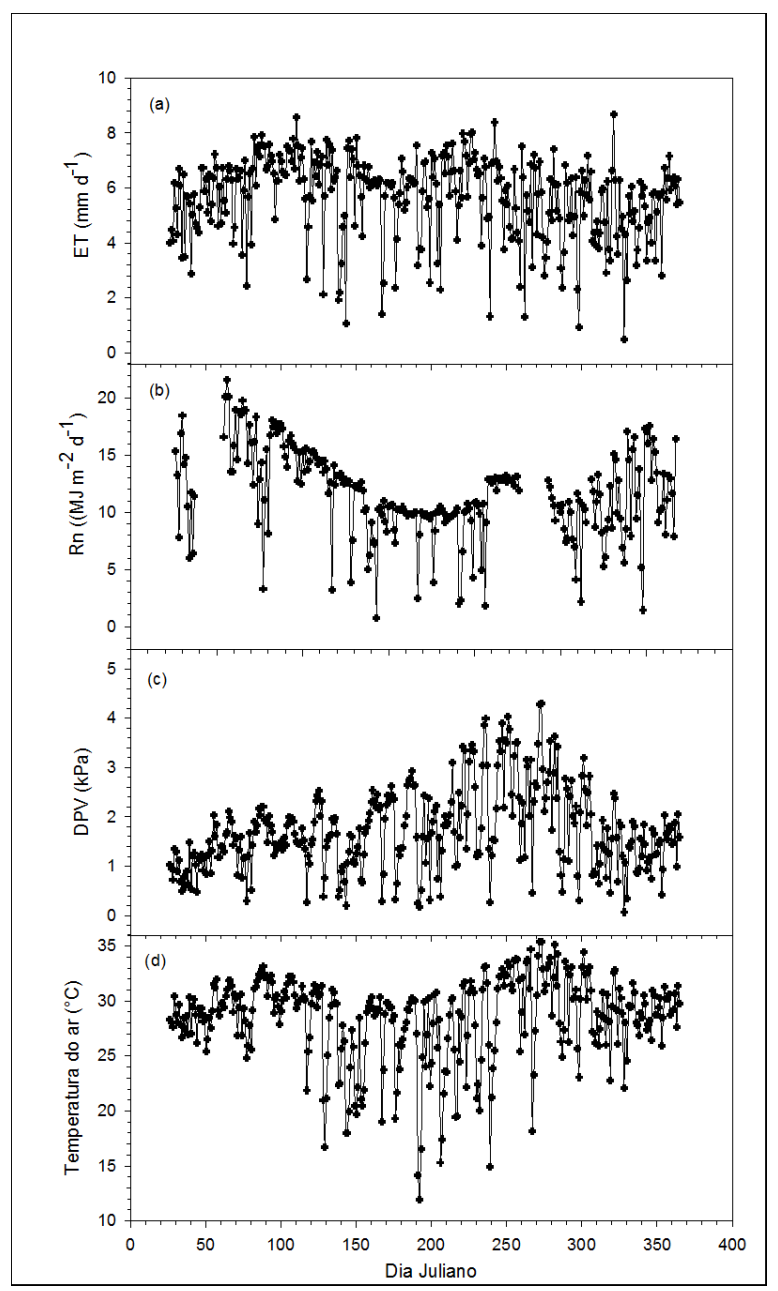

Figura 3-Média diária da (a) evapotranspiração estimada pelo método da razão de Bowen, (b) saldo de radiação, (c) déficit de pressão de vapor d'água e (d) temperatura do ar de janeiro a dezembro de 2007. 
as plantas de raízes superficiais (Meinzer et al., 1999). Por outro lado, maiores valores de evapotranspiração entre janeiro e maio estiveram relacionadas ao aumento da precipitação e da lâmina d'água de inundação.

A sazonalidade de evapotranspiração concorda com a sazonalidade do saldo de radiação que apresentou menores valores na estação seca (Figura 3b), aproximadamente $43 \%$ inferior que a estação úmida. A transição entre a estação úmida e seca coincide com a redução do saldo de radiação. O decréscimo do saldo de radiação não limitou a evapotranspiração e/ou a baixa demanda evaporativa, porque a área em estudo apresentou uma alta taxa de evapotranspiração devido a duração de 6 meses do ano de inundação (Figura 2).

O déficit de pressão de vapor (DPV) e a temperatura do ar apresentaram máximas e mínimas nas estações seca e úmida, respectivamente (Figuras $3 \mathrm{c}$ e $3 \mathrm{~d}$ ). A temperatura máxima do ar ocorreu em outubro, enquanto a temperatura mínima do ar ocorreu em julho (Figura 3d). Houve um aumento na temperatura do ar de $2,9 \%$ na estação seca em relação à estação úmida, representando em média $2,2^{\circ} \mathrm{C}$. A média mensal do déficit de pressão de vapor durante fevereiro foi $0,60 \mathrm{kPa}$ (estação úmida) e em setembro 2,2 kPa (estação seca) (Figura 3c).

A Figura 4 apresenta o perfil vertical da temperatura do ar no final da estação úmida (fevereiro a março), em médias horárias de $0 \mathrm{~h}, 2 \mathrm{~h}, 4 \mathrm{~h}$ e $21 \mathrm{~h}$ como período noturno, e $6 \mathrm{~h}, 9 \mathrm{~h}$, $15 \mathrm{~h}$ e $18 \mathrm{~h}$ como período diurno. Houve um padrão similar

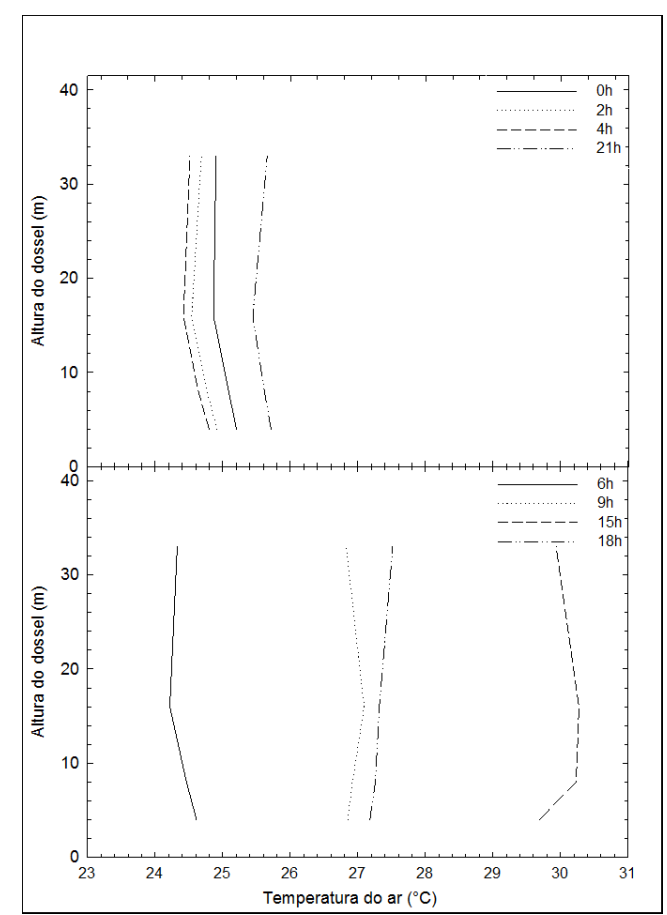

Figura 4-Médias horárias de temperatura do ar nas alturas 4, 8 e 16 m no (a) período noturno e (b) período diurno, estação úmida. durante o período noturno, com menor temperatura na altura de $16 \mathrm{~m}$ e um decréscimo de $1,34 \%$ em relação á temperatura a $4 \mathrm{~m}$ (as $0 \mathrm{~h}$ ), provavelmente porque na altura de $16 \mathrm{~m}$ havia uma maior densidade foliar, apresentando menor temperatura em função da maior umidade relativa devido às atividades de evapotranspiração. Aos 33 m, ocorreu um acréscimo de 0,14\% na temperatura em relação aos $16 \mathrm{~m}$. Ás $15 \mathrm{~h}$, a temperatura na altitude de 16 metros foi $1,96 \%$ e $1,12 \%$, maior do que as temperaturas á 4 e $33 \mathrm{~m}$, respectivamente.

A Figura 5 apresenta o perfil vertical da umidade relativa do ar no final da estação úmida (fevereiro a março), em médias horárias de $0 \mathrm{~h}, 2 \mathrm{~h}, 4 \mathrm{~h}$ e $21 \mathrm{~h}$ como período noturno e $6 \mathrm{~h}, 9 \mathrm{~h}$, $15 \mathrm{~h}$ e $18 \mathrm{~h}$ como período diurno. As $0 \mathrm{~h}$ a umidade do ar a $16 \mathrm{~m}$ foi de $1,99 \%$ e $6,69 \%$, e maior do que nas altitudes 4 e $33 \mathrm{~m}$, respectivamente. Ás $15 \mathrm{~h}$ a umidade a $4 \mathrm{~m}$ de altura foi $5,82 \% \mathrm{e}$ $4,25 \%$ maiores 16 e $33 \mathrm{~m}$, respectivamente. A umidade relativa do ar apresentou característica inversa a temperatura, pois os ambientes mais quentes são também os mais secos, e as áreas com cobertura arbórea apresentam umidade maior, devido à amenização térmica causada pelo dossel da mata.

\subsection{Coeficiente de Pristley-Taylor $(\alpha)$}

A Tabela 1 apresenta o coeficiente Priestley-Taylor estimado pela relação entre a ET e $(\mathrm{s} / \mathrm{s}+\gamma)(\mathrm{Rn}-\mathrm{G})$ apresentaram melhor coeficiente de correlação na estação seca. O coeficiente

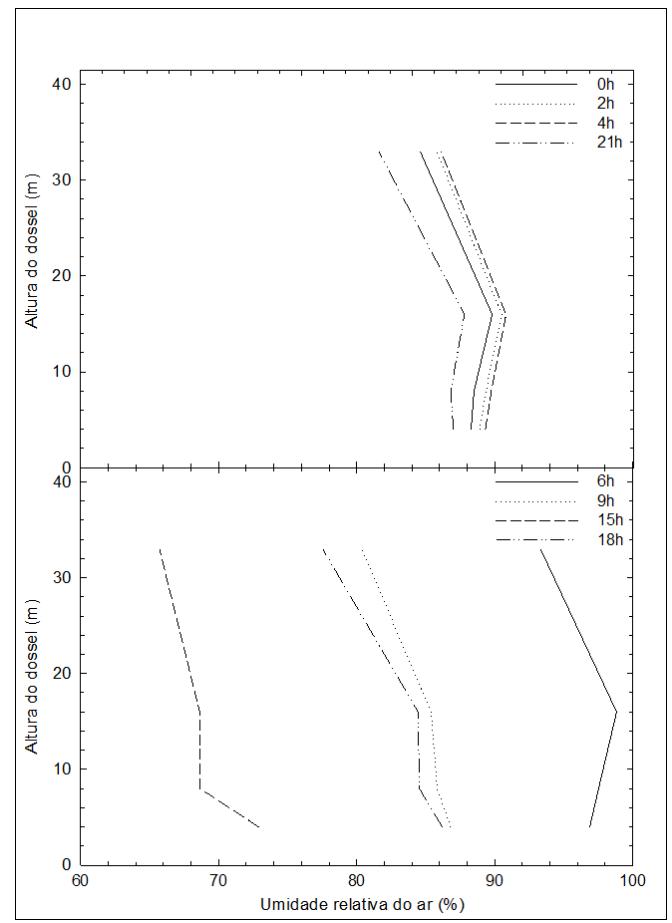

Figura 5 -Médias horárias de temperatura do ar nas alturas 4, 8 e $16 \mathrm{~m}$ no (a) período noturno e (b) período diurno, estação úmida. 
Tabela 1 - Estimativa do coeficiente Priestley-Taylor $(\alpha)( \pm$ evio padrão), calculado por meio da Equação 6 como a inclinação dos mínimos quadrados de regressão linear entre a evapotranspiração estimada pela razão de Bowen (variável dependente) e a (s/s $+\gamma)(\mathrm{Rn}-$ G) (variável independente).

\begin{tabular}{|c|c|c|c|c|}
\hline \multirow[b]{2}{*}{ Mêı } & \multicolumn{4}{|c|}{$a(+D P)$} \\
\hline & $E T\left(m m d^{-1}\right)$ & 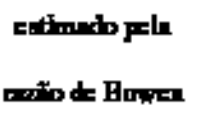 & $\boldsymbol{E}$ & $7^{2}$ \\
\hline Juncitu & $3,8 \pm 1,1$ & B,4 \pm D,12 & 6 & 842 \\
\hline Fa,crxtro & $4,0 \pm 1,3$ & $\mathbf{L ,}, 57 \pm \mathrm{B}, 15$ & 27 & 2,515 \\
\hline Mnga & $4,6 \pm 1,1$ & $\mathbf{B}, \mathbf{2} 2 \pm \mathrm{B}, 12$ & 3b & Q, 5 \\
\hline Abedl & $4,1 \pm 0,7$ & L, $62 \pm$ L, 13 & 31 & 846 \\
\hline Mnio & $2,7 \pm 0,8$ & $\mathbf{B}, \mathbf{1 3} \pm \mathbf{L}, \mathbf{3} \mathbf{n}$ & 31 & ge \\
\hline Junbo & $2,3 \pm 0,4$ & $\mathrm{~B}, \mathrm{BD} \pm \mathrm{B}, \mathrm{C9}$ & 3b & S13 \\
\hline Julle & $2,2 \pm 0,7$ & $\mathbf{L}, 97 \pm \mathrm{L}, \mathbf{2}$ & 31 & 872 \\
\hline Aqpm & $2,7 \pm 0,5$ & L,69 \pm L,11 & 3b & $\mathbf{9 1 2}$ \\
\hline Setembo & $2,1 \pm 0,7$ & $\mathrm{~B}, \mathrm{DL} \pm \mathrm{B}, 6$ & 23 & s90 \\
\hline Outnher & $3,1 \pm 1,3$ & L, 6 P \pm L,11 & 35 & Q51 \\
\hline Norambs & $3,6 \pm 1,3$ & D,34 \pm D, $\%$ & 3D & 677 \\
\hline Dex-mber & $4,4 \pm 1,1$ & $\mathrm{~B}, 49 \pm \mathrm{B}, 11$ & 31 & 8,44 \\
\hline
\end{tabular}

de Pristley-Taylor apresentou um comportamento padrão variando de 0,97 $\pm 0,52$ (julho) a 0,45 $\pm 0,12$ (janeiro) (Figura 6). A média do $\alpha$ na estação seca foi aproximadamente $50 \%$ maior do que na estação úmida.

Os valores de $\alpha$ foram menores do que mencionado por Priestley e Taylor (1972). De acordo com Priestley e Taylor (1972), $\alpha$ varia entre 1,08 (evaporação do solo nu) e 1,32 (evapotranspiração em vegetação densa) e usualmente emprega-se o valor de 1,26 em climas úmidos, entretanto estão de acordo com valores de $\alpha$ em floresta de transição Amazônia Cerrado (Vourlitis et al., 2002). Já em uma floresta tropical úmida, como a Amazônica em Manaus (Reserva Ducke), os valores de $\alpha$ variaram em função das condições de instabilidade e estabilidade da atmosfera nas horas do dia, variando de 0,67 a 1,16 e 1,28 a 3,12 , respectivamente, resultando em um $\alpha$ médio de 1,16 $\pm 0,56$ (Viswanadhama et al., 1991).

Maiores valores de $\alpha$ ocorreram quando os valores de saldo de radiação eram menores, com significativa correlação (correlação de $-0,80, p<0,05$ ), uma vez que os valores do saldo de radiação foram utilizados para determinação de $\alpha$. Houve

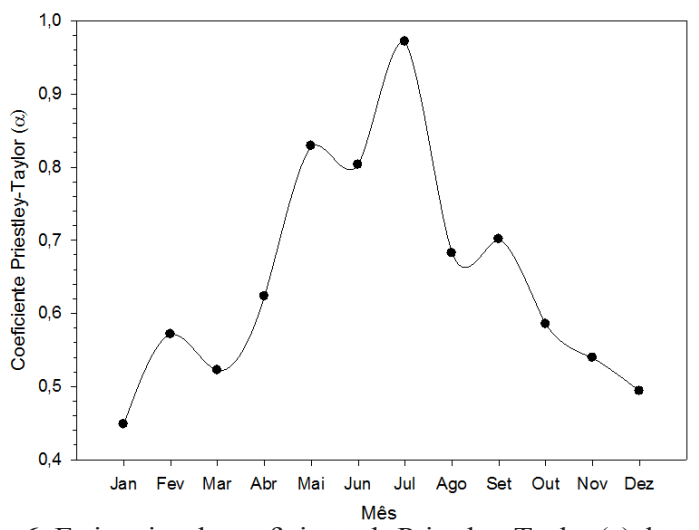

Figura 6 -Estimativa do coeficiente de Priestley-Taylor (a) determinado pelos mínimos quadrados, regressão linear entre evapotranspiração (estimado pela razão de Bowen) (variável dependente) e $(\mathrm{s} / \mathrm{s}+\mathrm{g})(\mathrm{Rn}-$ G) derivado de dados micrometeorológicos (variável independente). A linha sólida representa os valores interpolados de a utilizando uma função polinomial

também significativa correlação entre $\alpha$ e a temperatura do ar (correlação de $-0,65, \mathrm{p}<0,05$ ).

Da mesma forma, a diminuição do coeficiente de Priestley-Taylor durante a estação úmida, possivelmente em conseqüência do aumento do conteúdo de água no solo, em alguns meses, a água de inundação e/ou uma diminuição na demanda evaporativa (Figura 6), enquanto o saldo de radiação e a evapotranspiração diminuíram durante a estação seca.

\section{CONCLUSÕES}

Este artigo estimou o coeficiente Priestley-Taylor $(\alpha)$ por meio da evapotranspiração, estimada pelo método de Bowen para uma floresta monodominate Vochysia divergens, no Pantanal Mato-Grossense.

O coeficiente de Priestley-Taylor $(\alpha)$ variou de 0,53 (estação úmida) a 0,76 (estação seca), com média anual de 0,65, de acordo com o padrão climático da área em estudo, em função do aumento do conteúdo de água no solo/lâmina d'água de inundação e/ou diminuição na demanda evaporativa e altura do dossel.

\section{AGRADECIMENTOS}

Este estudo foi desenvolvido com auxílio financeiro do Conselho Nacional de Desenvolvimento Científico e Tecnológico (CNPq),pormeiodePesquisasEcológicas deLongaDuração(PELD) Pantanal Norte - Fluxos regionais e globais de massa e energia, registro 012/CAP/2006 e a Universidade Federal de Mato Grosso (UFMT).

\section{REFERÊNCIA BIBLIOGRÁFICA}

ARIEIRA, J.; NUNES DA CUNHA, C. Fitossociologia de uma 
floresta inundável monodominante de Vochysia divergens Pohl (Vochysiaceae), no Pantanal Norte, MT, Brasil. Acta Botanica Brasilica, v. 20, p. 569-580, 2006.

BOWEN, I. S. The ratio of heat losses by conductions and by evaporation from any water surface. Physical Review Serial, v. 2, n. 27, p. 779-787, 1926.

CASTELNOU, M. N.; FLORIANI, D.; VARGAS, I. A.; DIAS, J. B. Sustentabilidade socioambiental e diálogo de saberes: o Pantanal Mato-Grossense e seu espaço vernáculo como referência. Desenvolvimento e Meio Ambiente, n. 7, p. 41-67, 2003.

HARGREAVES, G. H. Defining and using reference evapotranspiration, J. Irrig. Drain. Eng., v. 120, p. 1132-1139, 199HASENACK, H.; CORDEIRO, J. L. P.; HOFMANN, G. S. O clima da RPPN SESC Pantanal. Porto Alegre, UFRGS, 27 p., 2003.

MEINZER, F. C., GOLdSTEIN, G.; FRANCO, A. C.; BUSTAMANTE, M.; IGLER, E.; JACKSON, P.; CALDAS, L.; RUNDEL, P. W. Atmospheric and hydraulic limitations on transpiration in Brazilian Cerrado woody species, Functional Ecology, 13, 273-282, 1999.

MIRANDA, A. C.; MIRANDA, H. S.; LLOYD J.; GRACE, J., FRANCEY, R. J.; MACINTRYRE, J. A.; MEIR, P.; RIGGAN, P.; LOCKWOOD, R. BRASS, J. Fluxes of carbon, water, and energy over Brazilian Cerrado: An analysis using eddy covariance and stable isotopes. Plant, Cell and Environment, 20, 315-328, 1997.

NUNES da CUNHA, C.; JUNK, W. J. Year-to-year changes in water level drive the invasion of Vochysia divergens in Pantanal glassland, Applied Vegetation Science, v. 7, p. 103-110, 2004.

PENHA, J. M.; DA SILVA, C. J.; BIANCHINI, I. Productivity of the aquatic macrophytes Pontederia lanceolata Nutt. (Pontederiaceae) on the floodplains of the Pantanal MatoGrossense, Brazil,. Wetlands Ecology and Management, 7/3, 155-163, 1999.

PENMAN, H. L. Natural evaporation from open water, bare soil, and grass. Proc. Roy. Soc., v. 193, p. 120-146, 1948.

PEREIRA, A. R.; VILLA NOVA, N. A.; SENTELHAS, P. C. O parâmetro de Priestley-Taylor para a estimativa da evapotranspiração de referência na escala mensal. Revista Brasileira de Agrometeorologia, v. 5, n. 1, p. 83-87, 1997.

PRIESTLEY, C. H. B.; TAYLOR, R. J. On the assessment of surface heat flux and evaporation, using large scale parameters. Monthly Weather Review, v. 100, n. 2, p.8192, 1972.

RAUPACH, M. R. Combination theory and equilibrium evaporation, Quart. J. Roy. Meteorol. Soc., v. 127, p. 1149-1181. 2001.

ROCHA, H. R.; MANZI, A. O.; CABRAL, O. M.; MILLER, S. D.; GOULDEN, M. L.; SALESKA, S. R.; R.-COUPE, N.; WOFSY, S. C.; BORMA, L. S.; ARTAXO, P.; VOURLITIS, G.; NOGUEIRA, J. S.; CARDOSO, F. L.; NOBRE, A. O D.; KRUIJT, B.; FREITAS, HELBER C.; VON RANDOW, C.; AGUIAR, R. G.; MAIA, J. F. Patterns of water and heat flux across a biome gradient from tropical forest to savanna in Brazil, Journal of Geophysical Research, v. 114, p. G00B12, 2009. doi:10.1029/2007JG000640.

SUMNER, D. M.; JACOBS, J. M. Utility of Penman-Monteith, Priestley-Taylor, reference evapotranspiration, and pan evaporation methods to estimate pasture evapotranspiration, Journal of Hydrology, v. 308, p. 81-104, 2005. doi:10.1016/j. jhydrol.2004.10.023.

THORNTHWAITE, C. W. An approach toward a rational classification of climate. Geogr. Rev, v. 38, p. 55-94, 1948. VISWANADHAMA, Y.; SILVA FILHO, V. P.; ANDRÉ, R. G. B The Priestley-Taylor parameter $\alpha$ for the Amazon Forest, Forest Ecology and Management, v. 38, 3-4, 211-225, 1991. doi:10.1016/0378-1127(91)90143-J

VOURLITIS, G. L.; PRIANTE-FILHO, N.; HAYASHI, M. M. S.; NOGUEIRA, J. S. DE; CASEIRO, F.; CAMPELO-JUNIOR J. H. Seasonal variations in the evapotranspiration of a transitional tropical forest of Mato Grosso, Brazil, Water Resour. Res.. v. 38, n. 6, p. 1094, doi:10.1029/2000WR000122.

XIAOYING, L.; ERDA, L. Performance of the Priestley-Taylor equation in the semiarid climate of North China. Agricultural Water Management, v. 71, p. 1-17, 2005. doi:10.1016/j. agwat.2004.07.007. 